\title{
S-unimodal Misiurewicz maps with flat critical points
}

\author{
by
}

\author{
Roland Zweimüller (London)
}

\begin{abstract}
We consider S-unimodal Misiurewicz maps $T$ with a flat critical point $c$ and show that they exhibit ergodic properties analogous to those of interval maps with indifferent fixed (or periodic) points. Specifically, there is a conservative ergodic absolutely continuous $\sigma$-finite invariant measure $\mu$, exact up to finite rotations, and in the infinite measure case the system is pointwise dual ergodic with many uniform and Darling-Kac sets. Determining the order of return distributions to suitable reference sets we obtain bounds on the decay of correlations and on wandering rates. Assuming some control of the local behaviour of $T$ at $c$, we show that in most cases, e.g. whenever the postcritical orbit has a Lyapunov exponent, the tail of the return distribution is in fact regularly varying, which implies various distributional limit theorems.
\end{abstract}

1. Introduction. While the dynamics of one-dimensional dynamical systems with nonflat critical points has been the object of intense study, it seems that little attention has been paid to the case of flat tops (i.e. critical points where all derivatives of the map vanish). The only references I am aware of are $[\mathrm{BM}]$ and $[\mathrm{Th}]$. The purpose of the present note is to point out that maps with flat tops do have some very interesting ergodic properties when regarded as nonsingular transformations with respect to Lebesgue measure $\lambda$ and can in fact be interpreted as generalizations of transformations with indifferent fixed (or periodic) points. The latter class of systems has recently attracted a lot of attention. In the probability preserving situation, their slow mixing behaviour and its probabilistic consequences have been investigated by several authors (see e.g. [LSV], [Hu], [Yo], [Sa], [Ho], or [Z2]), while the infinite measure case constitutes one of the most popular families of examples in infinite ergodic theory (cf. [A0], [A2], [T1]-[T4], [Z1]). Here we advertise maps with flat tops as a class of systems which considerably extends the supply of examples exhibiting these features. The reason

2000 Mathematics Subject Classification: Primary 28D05, 37A25; Secondary 37A40, 37E05, 60F05.

Key words and phrases: neutral orbit, slow mixing, infinite invariant measure, central limit theorem, stable limit law, Lyapunov exponent. 
for their weak hyperbolicity is not as obvious as in the case of neutral fixed points, which of course makes them even more interesting. Roughly speaking, the critical point has a (usually nonperiodic) "indifferent orbit" along which the expansion of the system is very poor.

Throughout, $\lambda$ will denote one-dimensional Lebesgue measure, and $\mathcal{B}$ will be the Borel $\sigma$-field of the space under consideration. We consider $S$ unimodal interval maps $T:[a, b] \rightarrow[a, b]$, i.e. $\mathcal{C}^{3}$ maps with a unique critical point $c \in(a, b), T^{\prime} c=0$, such that $T a=T b=a$, for which the Schwarzian derivative $\mathcal{S} T:=T^{\prime \prime \prime} / T^{\prime}-\frac{3}{2}\left(T^{\prime \prime} / T^{\prime}\right)^{2}$ is nonpositive (with $\mathcal{S} T(c)=-\infty$ ). The critical point is said to be flat if $T^{(n)}(c)=0$ for all $n \geq 1$. A map $T$ is said to be a Misiurewicz map if there is some open interval $I_{\mathcal{M}}$ around $c$ such that $c_{n}:=T^{n} c \notin I_{\mathcal{M}}$ for $n \geq 1$, and if $T$ has no sinks (open intervals homeomorphically mapped into themselves by some iterate of $T$ ). It is well known that full families of unimodal maps contain uncountably many Misiurewicz maps (see e.g. the combinatorial characterization we use in Section 5 below). In the present paper we shall restrict our attention to the Misiurewicz case, which is a natural nontrivial starting point for an investigation of finer ergodic properties of unimodal maps with flat tops. Of course, one should expect suitably weakened conditions on the recurrence behaviour of the critical orbit to be sufficient for the results to follow, but it seems worthwile to expose the effects of flat tops in a setup with limited technical difficulties.

EXAmple 1 (A basic family of examples). Fix any $p>0, s>p+1$, and define $T_{a}=T_{a,(p, s)}:[-1,1] \rightarrow[-1,1], a \in(0,1]$, by

$$
T_{a}(x):=2 a \cdot\left(1-e^{s\left(1-|x|^{-p}\right) / p}\right)-1 .
$$

We shall see below that the Misiurewicz maps from this family in many respects resemble the behaviour of maps with indifferent fixed points $x_{T}$ of the form $T x=x+$ const $\cdot\left(x-x_{T}\right)^{1+p}+o\left(\left(x-x_{T}\right)^{1+p}\right)$ near $x_{T}$.

As a warm-up, we will show in the next section that systems of this type always have $\sigma$-finite absolutely continuous invariant measures (acims):

Theorem 1 (Invariant measures for Misiurewicz maps). Every S-unimodal Misiurewicz map $T$ is ergodic with respect to $\lambda$ and has a conservative $\sigma$-finite invariant Borel measure $\mu \ll \lambda$. The measure $\mu$ is finite iff $\int \log \left|T^{\prime}\right| d \lambda>-\infty$.

By ergodicity, $\mu$ is unique up to a constant factor, and Lebesgue a.e. point is eventually mapped into the support of $\mu$. If $\mu$ is finite, we will always assume it to be normalized.

REMARK 1. Existence and basic properties of finite absolutely continuous invariant measures for (not necessarily unimodal) Misiurewicz maps 
with nonpositive Schwarzian derivative, and $\log \left|T^{\prime}\right| \in L_{1}(\lambda)$ (also shown to be a necessary condition in the presence of the others), have been proved in $[\mathrm{BM}]$. Needless to say, we will repeatedly use arguments similar to those of $[\mathrm{Mi}]$ and $[\mathrm{BM}]$. In [Th] even a Jakobson-type result for certain families of S-unimodal maps with flat tops and $\log \left|T^{\prime}\right| \in L_{1}(\lambda)$ (including the family of Example 1 for $p<1 / 8)$ is established.

EXAMPLE 2 (Continuation of Example 1). The Misiurewicz maps $T=T_{a}$ from the family of Example 1 preserve a probability measure $\mu \ll \lambda$ provided that $p<1$. For $p \geq 1$ the acim $\mu$ is infinite, but still conservative ergodic.

Below we are going to explore some finer ergodic properties of the measure preserving systems thus obtained.

\section{Preliminaries on the Schwarzian derivative, piecewise mono-} tonic and induced systems. Proof of Theorem 1. We recall some important concepts, and collect a few fundamental results that will be used subsequently. Although we use (almost) the same notations and conventions as in [Z1], we include the relevant definitions for the reader's convenience.

The Schwarzian derivative and the Koebe principle. Recall first that $\mathcal{S} T \leq 0$ implies $\mathcal{S}\left(T^{n}\right) \leq 0$ for all $n \geq 1$. As observed in [Mi], a $\mathcal{C}^{3}$ function $T$ which has no critical point in an open interval $J$ satisfies $\mathcal{S} T \leq 0$ on $J$ iff $1 / \sqrt{\left|T^{\prime}\right|}$ is convex on $J$. Let $I \subseteq J$ be a subinterval; then $J$ is said to contain a $\delta$-scaled neighbourhood of $I$ if $J \backslash I$ consists of two components, each of length at least $\delta \lambda(I)$. The above characterization of $\mathcal{S} T \leq 0$ implies the all important Koebe principle providing us with good distortion control:

Let $I \subseteq J$ be open intervals and assume that $T \in \mathcal{C}^{3}(J)$ satisfies $\mathcal{S} T \leq 0$ and has no critical point in $J$. If $T(J)$ contains a $\delta$-scaled neighbourhood of $T(I)$, then

$$
\sup _{x, y \in I}\left|\frac{T^{\prime} x}{T^{\prime} y}\right| \leq\left(\frac{1+\delta}{\delta}\right)^{2} .
$$

Piecewise monotonic systems. A piecewise monotonic system is a triple $(X, T, \xi)$, where $X$ is the disjoint union of some countable family $\xi_{0}$ of open intervals, $\xi$ is a collection of nonempty pairwise disjoint open subintervals (the cylinders of rank one) with $\lambda(X \backslash \bigcup \xi)=0$, and $T: X \rightarrow X$ is a map such that $\left.T\right|_{Z}$ is continuous and strictly monotonic for each $Z \in \xi$. Given such a system, we let $\xi_{n}$ denote the family of cylinders of rank $n$, that is, the nonempty sets of the form $Z=\left[Z_{0}, \ldots, Z_{n-1}\right]:=\bigcap_{i=0}^{n-1} T^{-i} Z_{i}$ with $Z_{i} \in \xi$. We let $V_{Z}:=\left(\left.T^{n}\right|_{Z}\right)^{-1}$ be the inverse of the branch $\left.T^{n}\right|_{Z}$. We denote by $\partial \xi$ the collection of endpoints of members of $\xi$. The fundamental partition $\xi$ and the system $(X, T, \xi)$ are said to be Markov if $T Z \cap Z^{\prime} \neq \emptyset$ 
implies $Z^{\prime} \subseteq T Z$ whenever $Z, Z^{\prime} \in \xi$. In this case the image partition $T_{*} \xi$ (i.e. the coarsest partition into intervals with respect to which each $T Z$, $Z \in \xi$, is measurable) is coarser than $\xi$. We write $\xi_{n}(x)$ for the member of $\xi_{n}$ containing the point $x$. If $x$ is the common endpoint of two cylinders from $\xi_{n}$, we denote them by $\xi_{n}\left(x^{-}\right)$and $\xi_{n}\left(x^{+}\right)$respectively.

First-return maps and induced systems. Let $T$ be a nonsingular transformation of some $\sigma$-finite measure space $(X, \mathcal{B}, \lambda)$. Consider a recurrent set $Y \in \mathcal{B}$, i.e. one for which $Y \subseteq \bigcup_{n \geq 1} T^{-n} Y \bmod \lambda$. (If in fact $X=$ $\bigcup_{n \geq 1} T^{-n} Y \bmod \lambda$, then $Y$ is called a sweep-out set.) Then the first return time function $\varphi_{Y}$ given by $\varphi_{Y}(x):=\min \left\{n \geq 1: T^{n} x \in Y\right\}$ is finite a.e. We define the induced or first-return map $T_{Y}: Y \rightarrow Y \bmod \lambda$ by $T_{Y} x:=T^{\varphi_{Y}(x)} x$. The $n$th return time on $Y$ is then given by

$$
\varphi_{Y, n}(x):=\sum_{k=0}^{n-1} \varphi_{Y}\left(T_{Y}^{k} x\right) .
$$

If $\mu$ is a measure on $\mathcal{B}$ with $0<\mu(Y)<\infty$, we let $\mu_{Y}$ denote its normalized restriction to $Y: \mu_{Y}(A):=\mu(Y)^{-1} \mu(A \cap Y)$. Generally, objects associated with the induced map will notationally be identified by the subscript $Y$. We will repeatedly use the well known intimate relation between the basic ergodic properties of $T$ and $T_{Y}$ (see e.g. [T2] or Lemma 1 of [Z1]).

Suppose that $(X, T, \xi)$ is a piecewise monotonic system and consider some recurrent set $Y \subseteq X$. Assume that $Y$ is the union of some finite family $\xi_{Y, 0}$ of disjoint open subintervals of $X$, measurable $\xi \bmod \lambda$. We define the induced partition of $\xi$ on $Y$ to be

$$
\xi_{Y}:=\bigcup_{n \geq 1}\left\{\{\varphi=n\} \cap Z \cap T^{-n} M: Z \in \xi_{n}, M \in \xi_{Y, 0}\right\} .
$$

We see that $T_{Y}$ is piecewise monotonic, and $\xi_{Y}$ is its natural partition into intervals on which it is continuous and monotonic. We shall call $\left(Y, T_{Y}, \xi_{Y}\right)$ the system which $(X, T, \xi)$ induces on $Y$.

Some basic properties of $S$-unimodal maps. Henceforth $T:[a, b] \rightarrow[a, b]$ will be some S-unimodal map with critical point $c$. If $T$ has no sinks, Theorem 1.2 of [Mi] implies that

$$
\text { the partition } \xi=\{(a, c),(c, b)\} \text { generates. }
$$

Also, $T$ has good expansion properties as long as we stay away from the critical point: The proof of Theorem 1.3 of [Mi] shows that

(3) for any neighbourhood $J$ of the critical point $c$ there are $\varrho>1$ and $K>0$ such that for all $x \in[a, b]$ and $n \geq 1$, from $x, T x, \ldots, T^{n-1} x \notin J$ it follows that $\left|\left(T^{n}\right)^{\prime}(x)\right|>K \cdot \varrho^{n}$. 
Together with a standard distortion argument this reveals that

$$
\lambda\left(\bigcap_{k=0}^{n-1} T^{-k} J^{c}\right) \text { is exponentially small in } n,
$$

and in particular that

$$
\text { every neighbourhood } J \text { of } c \text { is recurrent and sweeps the space. }
$$

These observations are enough to prove the existence of a unique conservative ergodic $\sigma$-finite acim for Misiurewicz maps by inducing.

Definition 1. Given an S-unimodal Misiurewicz map $T$, we fix as a reference set the interval $Y=Y(T):=\xi_{\bar{n}}\left(c^{-}\right) \cup\{c\} \cup \xi_{\bar{n}}\left(c^{+}\right) \subset I_{\mathcal{M}}$ where $\bar{n} \in \mathbb{N}$ is so large that $\bar{\delta}:=\operatorname{dist}\left(Y, I_{\mathcal{M}}^{\mathrm{c}}\right)>0$ and $\operatorname{dist}\left(T Y, I_{\mathcal{M}}\right)>0$ (recall $(2))$.

Proof of Theorem 1. By (5), it is possible to induce on $Y=Y(T)$, and we claim that

the induced system $\left(Y, T_{Y}, \xi_{Y}\right)$ is piecewise surjective and has bounded distortion, i.e. there is some $\delta_{\mathcal{M}}>0$ such that

$$
\sup _{\substack{n \geq 1 \\ x, y \in W \in \xi_{Y}}}\left|\frac{\left(T^{n}\right)^{\prime}(x)}{\left(T^{n}\right)^{\prime}(y)}\right| \leq\left(\frac{1+\delta_{\mathcal{M}}}{\delta_{\mathcal{M}}}\right)^{2} .
$$

It is straightforward to check that $\left(Y, T_{Y}, \xi_{Y}\right)$ is piecewise onto. Moreover, each branch $\left.T_{Y}\right|_{W}, W \in \xi_{Y}$, is the restriction to $W$ of some branch $\left.T^{k}\right|_{Z}$, $Z \in \xi_{k}$, of $T$ with image $T^{k} Z$ covering $I_{\mathcal{M}}$ and hence containing a $\delta_{\mathcal{M}^{-}}$ scaled neighbourhood of $Y=T_{Y} W$, where $\delta_{\mathcal{M}}:=\bar{\delta} / \lambda(Y)$ does not depend on $W$. By the Koebe principle (1), the induced system therefore has bounded distortion.

Hence some iterate of $T_{Y}$ is uniformly expanding and, by folklore results, $T_{Y}$ is exact and preserves a probability measure $\mu_{Y} \ll \lambda$ with Lipschitz continuous density $h_{Y}$ satisfying $\log h_{Y} \in L_{\infty}(Y)$. By standard results (see e.g. Lemma 1 of [Z1], or [T2]), $\mu_{Y}$ extends to some $\sigma$-finite $T$-invariant measure $\mu \ll \lambda$ on $X$, and since $Y$ sweeps $X$ (cf. (5)), there are no other acims for $T$. The characterization of finiteness of $\mu$ is immediate from Theorems 1 and 4 of $[\mathrm{BM}]$ (see also Remark 3 below).

REMARK 2. Applying the Koebe principle relative to $I_{\mathcal{M}}$ to small intervals $(x, y)$ in $Y$ shows that the induced system $\left(Y, T_{Y}, \xi_{Y}\right)$ in $(6)$ in fact has the Gibbs property in the sense of [A0], [AD1].

\section{Return distributions, decay of correlations, and a central} limit theorem. In the Misiurewicz case it is easy to determine the order of the tail of the return distribution to our reference set $Y=Y(T) \subset I_{\mathcal{M}}$. 
To formalize this, we need a few more notations. Let $T$ be some S-unimodal Misiurewicz map and recall that $V_{Z_{0}}=\left(\left.T\right|_{Z_{0}}\right)^{-1}$ and $V_{Z_{1}}=\left(\left.T\right|_{Z_{1}}\right)^{-1}$ denote the two inverse branches of $T$. Define $U(x):=c_{1}-T(x-c), x \in[a-c, b-c]$, and $V_{i}:\left[0, c_{1}-a\right] \rightarrow[0, \infty)$ as $V_{i}(y):=\left|V_{Z_{i}}\left(c_{1}-y\right)-c\right|$, which transforms the singularity to the origin, so that the behaviour of the $V_{i}$ at zero describes the type of the critical point. (Of course, if $T$ is symmetric about $c$, then $V_{0}=V_{1}$.) Since $\mathcal{S} T \leq 0$, there is some $\delta_{V}>0$ such that the $V_{i}$ are strictly concave on $\left[0, \delta_{V}\right]$,

(cf. Lemma 3 of $[\mathrm{BM}]$ ). We let $V:=V_{0}+V_{1}$.

Proposition 1 (Asymptotic order of the return distributions). Let $T$ be an S-unimodal Misiurewicz map. There are constants $K_{l}, K_{u}>0$ and $q_{l}, q_{u} \in(0,1)$ such that for $n$ sufficiently large,

$$
K_{l} \cdot V\left(q_{l}^{n}\right) \leq \mu_{Y}\left(Y \cap\left\{\varphi_{Y}>n\right\}\right) \leq K_{u} \cdot V\left(q_{u}^{n}\right) .
$$

In particular, if $c$ is flat, $\mu_{Y}\left(Y \cap\left\{\varphi_{Y}>n\right\}\right)$ does not decrease exponentially fast.

Proof. We have $Y \cap\left\{\varphi_{Y}>n\right\}=Y \cap T^{-1}\left(\bigcap_{j=0}^{n-1} T^{-j} Y^{\mathrm{c}}\right), n \geq 1$. As $\mathcal{S} T \leq 0$ and $T^{\prime}(c)=0$, there is some $\varepsilon>0$ such that for any set $M$ with $\lambda(M)<\varepsilon$ we have

$$
\lambda\left(Z_{i} \cap T^{-1} M\right) \leq \lambda\left(Z_{i} \cap T^{-1}\left(c_{1}-\lambda(M), c_{1}\right)\right)=V_{i}(\lambda(M)), \quad i \in\{0,1\} .
$$

Recalling (4) we therefore see that there is some $q_{u} \in(0,1)$ such that for $n$ sufficiently large,

$$
\lambda\left(Z_{i} \cap\left\{\varphi_{Y}>n\right\}\right) \leq V_{i}\left(\lambda\left(\bigcap_{j=0}^{n-1} T^{-j} Y^{\mathrm{c}}\right)\right) \leq V_{i}\left(q_{u}^{n}\right) .
$$

Since the invariant density $h=d \mu / d \lambda$ is bounded away from 0 and $\infty$ on $Y$, the upper estimate follows. To prove the lower estimate, consider the subset of $\bigcap_{j=0}^{n-1} T^{-j} Y^{\mathrm{c}}$ consisting of those points which closely follow the critical orbit all along, i.e. $M_{n}:=\bigcap_{j=0}^{n-1} T^{-j}\left\{x:\left|x-c_{j+1}\right|<\bar{\delta}\right\}, n \geq 1$, where $\bar{\delta}:=\operatorname{dist}\left(Y, I_{\mathcal{M}}^{\mathrm{c}}\right)>0$ as in the proof of Theorem 1 . The $M_{n}$ are intervals adjacent to $c_{1}$, and therefore $\lambda\left(Z_{i} \cap T^{-1} M_{n}\right)=V_{i}\left(\lambda\left(M_{n}\right)\right)$. However, as $T^{\prime}$ is bounded, $\lambda\left(M_{n}\right)$ cannot decrease faster than at some fixed exponential rate, and the argument is completed as before. Finally, if the critical point is flat, we have $x^{r}=o(V(x))$ as $x \rightarrow 0$ for every $r \in(0,1)$, and hence $\gamma^{n}=o\left(V\left(q^{n}\right)\right)$ as $n \rightarrow \infty$ for any $\gamma, q \in(0,1)$.

REMARK 3 (Finiteness of the invariant measure). Since in any case we have $0<\mu(Y)<\infty$, Kac's formula ensures that $\mu$ is finite iff the return time distribution of $Y$ has finite expectation, that is, iff $\sum_{n>0} \mu_{Y}(Y \cap$ $\left.\left\{\varphi_{Y}>n\right\}\right)<\infty$. Together with (7), Lemmas 1 and 2 of $[\mathrm{BM}]$ and our 
proposition show that the latter is equivalent to integrability of any of the functions $\log \left|T^{\prime}\right|$ and $\log |T-T(c)|$. We thus recover Theorem 4 of [BM] using the existence of a unique $\sigma$-finite acim rather than their Lemma 11.

ExAmple 3 (Continuation of Example 1). Fix $p>0, s>p+1, a \in(0,1]$, and consider $T:=T_{a}$. Then

$$
V_{i}(y)=\left(-\frac{p}{s} \log \left(\frac{y}{2 a e^{s / p}}\right)\right)^{-1 / p}, \quad i \in\{0,1\},
$$

so that for any $K>0$ and $q \in(0,1), V_{i}\left(K q^{n}\right) \sim[p(-\log q) / s]^{-\alpha} n^{-\alpha}$ as $n \rightarrow \infty$, where $\alpha:=p^{-1}$. Consequently, $\mu_{Y}\left(Y \cap\left\{\varphi_{Y}>n\right\}\right) \approx n^{-\alpha}$.

Together with the observation (6), these bounds on the tail weights enable us at once to apply the results on mixing rates for towers above well mixing transformations established in [Yo]. We let $\mathbf{P}: L_{1}(\lambda) \rightarrow L_{1}(\lambda)$ denote the transfer (or Perron-Frobenius) operator of $T$ with respect to $\lambda$, which is characterized by

$$
\int \mathbf{P} u \cdot g d \lambda=\int u \cdot(g \circ T) d \lambda
$$

for $u \in L_{1}(\lambda)$ and $g \in L_{\infty}(\lambda)$, and extends to quasi-integrable functions $u$ by a straightforward approximation procedure. Given an interval $I$, we let $\mathcal{H}(I)$ denote the family of (uniformly) Hölder continuous functions on $I$.

If $\nu$ is a probability measure on the measurable space $(X, \mathcal{A})$ and $\left(R_{n}\right)_{n \geq 1}$ is a sequence of measurable real functions on $X$, distributional convergence of $\left(R_{n}\right)_{n \geq 1}$ with respect to $\nu$ to some random variable $R$ will be denoted by $R_{n} \stackrel{\nu}{\Longrightarrow} R$. Strong distributional convergence $R_{n} \stackrel{\mathcal{L}(\lambda)}{\Longrightarrow} R$ on the $\sigma$-finite measure space $(X, \mathcal{A}, \lambda)$ means that $R_{n} \stackrel{\nu}{\Longrightarrow} R$ for all probability measures $\nu \ll \lambda$. If $T$ is a nonsingular ergodic transformation on $(X, \mathcal{A}, \lambda)$, a compactness property implies that if $R_{n} \circ T-R_{n} \stackrel{\lambda}{\longrightarrow} 0$, then $R_{n} \stackrel{\mathcal{L}(\lambda)}{\Longrightarrow} R$ as soon as $R_{n} \stackrel{\nu}{\Longrightarrow} R$ for some $\nu \ll \lambda$ (compare Section 3.6 of [A0], or [A1]). Specifically, this applies if $T$ is not completely dissipative and $R_{n}=b_{n}^{-1} \mathbf{S}_{n}(f)=b_{n}^{-1} \sum_{k=0}^{n-1} f \circ T^{k}, n \in \mathbb{N}$, for some $f \in L_{1}(\lambda)$ and suitable normalization $\left(b_{n}\right)_{n \geq 1}$.

THEOREM 2 (Rates of mixing, decay of correlations, and CLT). Let T be an S-unimodal Misiurewicz map. Assume that $\mathcal{V}(t):=V\left(e^{-t}\right)=O\left(t^{-\alpha}\right)$ for some $\alpha>1$ (hence $\mu$ is finite).

(a) For any probability density $u \in \mathcal{H}(Y)$,

$$
\left\|\mathbf{P}^{n} u-h\right\|_{L_{1}(\lambda)}=O\left(n^{1-\alpha}\right) \quad \text { as } n \rightarrow \infty,
$$

and for any $f \in L_{\infty}(\lambda)$ and $g \in \mathcal{H}(Y)$,

$$
\left|\int f\left(g \circ T^{n}\right) d \mu-\mu(f) \mu(g)\right|=O\left(n^{1-\alpha}\right) \quad \text { as } n \rightarrow \infty .
$$


(b) If $\alpha>2$, then for any $f \in \mathcal{H}(Y)$,

$$
\frac{1}{\sqrt{n}} \sum_{k=0}^{n-1}\left(f \circ T^{k}-\mu(f)\right) \stackrel{\mathcal{L}(\lambda)}{\Longrightarrow} \mathcal{N}(0, \sigma(f)),
$$

with $\sigma(f)>0$ iff $f \neq g \circ T-g$ for $g$ measurable.

ExAmple 4 (Continuation of Example 1). Recalling Example 3, we see that $n \mathcal{V}(n) \sim$ const $\cdot n^{1-\alpha}$, and the CLT applies if $p<1 / 2$.

Proof of Theorem 2. According to our assumption on $\mathcal{V}$, Proposition 1 implies $\mu_{Y}\left(Y \cap\left\{\varphi_{Y}>n\right\}\right) \approx v(t)$. We apply the abstract mixing theorm of [Yo]. The induced system $\left(Y, T_{Y}, \xi_{Y}\right)$ on our reference set $Y=Y(T)$ is Rényi and hence satisfies the assumptions on the return map $F^{R}$ in the abstract framework of $[\mathrm{Yo}]$. The results of this paper are formulated for the Kakutani tower $(Z, S)$ above $\left(Y, T_{Y}\right)$ with height function $\varphi$, i.e. $Z:=\{(y, k) \in Y \times \mathbb{N}$ : $k \leq \varphi(y)\}$ and $S(y, k)=(y, k+1)$ if $k<\varphi(y)$ and $S(y, \varphi(y))=\left(T_{Y} y, 1\right)$. As this tower is always injective outside its roof $\{(y, \varphi(y)) \in Z\}$, it does not represent the general system $(X, T)$ with induced map $\left(Y, T_{Y}\right)$ and return time $\varphi$. However, it is easily seen that the bounds on the rates of mixing and the CLT apply to any such system, since in an obvious way $(X, T)$ is always a factor of $(Z, S)$.

REMARK 4. Theorem 2 of [Sa] shows that in many situations the bounds given in [Yo] are sharp if $\mu_{Y}\left(Y \cap\left\{\varphi_{Y}>n\right\}\right) \approx n^{-\alpha}$ with $\alpha>2$. This, too, applies in our setup, since it is easily seen that the return time function $\varphi$ is aperiodic.

REMARK 5. The extension of Young's results to the case of slowly varying orders given in $[\mathrm{Ho}]$ also applies in our situation.

\section{Tail field, pointwise dual ergodicity, uniform convergence,} and Darling-Kac sets. Recall that $T$ is said to be exact with respect to $\lambda$ if for any set $A$ from its tail $\sigma$-field $\mathcal{B}_{\infty}:=\bigcap_{n \geq 0} T^{-n} \mathcal{B}$ either $A$ or $A^{\text {c }}$ has zero Lebesgue measure. Below we shall prove

Theorem 3 (Finite tail field). S-unimodal Misiurewicz maps $T$ are Lebesgue exact up to a finite rotation.

The main result of the present section, which (though not void in the finite measure case) is mainly of interest in the infinite measure preserving situation, concerns the asymptotic behaviour of the transfer operator. If $\mu$ is infinite, then exactness implies that $\lim _{n \rightarrow \infty} \int_{A} \mathbf{P}^{n} u d \lambda=0$ for all $u \in$ $L_{1}(\lambda)$ and $A \in \mathcal{B}$ with $\mu(A)<\infty$ (cf. [T3]). It is then natural to consider suitably normalized iterated densities in order to compensate this escape of mass. Again, we obtain a result which is analogous to what we know about 
transformations with indifferent fixed points (cf. [T3] and Theorem 9 of [Z1]). In the latter situation the obvious family of good sets of finite measure consists of those which are bounded away from the neutral fixed points. Hardly surprising, the collection $\mathcal{E}(T):=\left\{M \subseteq X: \operatorname{dist}\left(M,\left\{c_{n}\right\}_{n \geq 1}\right)>0\right\}$ plays a similar role in the present setup. Notice that by boundedness of $X$, any set $A \in \mathcal{E}(T)$ is contained in some finite union of intervals from $\mathcal{E}(T)$.

TheOREM 4 (Uniform convergence of averaged iterated densities). Let $T$ be an S-unimodal Misiurewicz map. Then the invariant density has a version $h$ with $h \in \mathcal{D}(I) \cap L_{\infty}(\lambda)$ for all intervals $I \in \mathcal{E}(T)$, and there is some sequence $\left(a_{n}(T)\right)_{n \geq 1}$ in $\mathbb{R}_{+}\left(a_{n}=n\right.$ if $\mu$ is finite, and $a_{n}=o(n)$ otherwise) such that for any Riemann-integrable function $u$,

$$
\frac{1}{a_{n}(T)} \sum_{k=0}^{n-1} \mathbf{P}^{k} u \rightarrow \lambda(u) \cdot h \quad \text { as } n \rightarrow \infty,
$$

uniformly on members of $\mathcal{E}(T)$.

A conservative ergodic measure preserving transformation (cempt) $T$ on a $\sigma$-finite space $(X, \mathcal{B}, \mu)$ is called pointwise dual ergodic (cf. [A1] or Section 3.7 in [A0]) if there are positive constants $a_{n}(T), n \geq 1$, such that

$$
\frac{1}{a_{n}(T)} \sum_{k=0}^{n-1} \mathbf{T}^{k} f \rightarrow \mu(f) \quad \text { a.e. as } n \rightarrow \infty \text { for all } f \in L_{1}(\mu),
$$

where $\mathbf{T}: L_{1}(\mu) \rightarrow L_{1}(\mu)$ is the dual operator of $T$, that is, the transfer operator with respect to the invariant measure $\mu$, characterized by $\int_{X} \mathbf{T} u$. $f d \mu=\int_{X} u \cdot f \circ T d \mu$ for $u \in L_{1}(\mu), f \in L_{\infty}(\mu)$. (By Hurewicz's ergodic theorem, cf. [A0, Section 2.2], this holds as soon as the convergence in (8) takes place for one function $f \in L_{1}(\mu), \mu(f) \neq 0$.) The sequence $\left(a_{n}(T)\right)_{n \geq 1}$ which is then uniquely determined up to asymptotic equivalence is called the return sequence of $T$. We let $\mathcal{U}(T)$ denote the family of uniform sets, i.e. those $B \in \mathcal{B}$ on which $a_{n}^{-1} \sum_{k=0}^{n-1} \mathbf{T}^{k} f$ converges uniformly $(\bmod \mu)$ for some $f \in L_{1}^{+}(\mu)$.

Whenever $T$ is a cempt of $(X, \mathcal{B}, \mu)$, a set $B \in \mathcal{B}$ with $0<\mu(B)<\infty$ is called a Darling-Kac set if for some sequence $\left(a_{n}\right)$ in $\mathbb{R}_{+}, a_{n}^{-1} \sum_{k=0}^{n-1} \mathbf{T}^{k} 1_{B}$ converges uniformly $(\bmod \mu)$ on $B$. The mere existence of Darling-Kac sets is a rather strong qualitative mixing property for infinite measure preserving transformations, and in particular implies pointwise dual ergodicity (with return sequence $\left(a_{n}\right)$, cf. Proposition 3.7.5 of [A0]).

As the invariant density $h$ of $T$ is bounded away from zero and infinity on members of $\mathcal{E}(T)$, and $\mathbf{T} u=h^{-1} \mathbf{P}(h u)$, Theorem 4 implies:

Corollary 1 (Pointwise dual ergodicity, uniform sets, and DK sets). Let $T$ be an S-unimodal Misiurewicz map. Then $T$ is pointwise dual ergodic 
with $\mathcal{E}(T) \subseteq \mathcal{U}(T)$, and any $B \in \mathcal{E}(T)$ satisfying $\lambda(\partial B)=0$ is a Darling-Kac set for $T$.

According to Theorem 10 of [Z1], the collection of Darling-Kac sets cannot be too large, which also shows that there is no way to extend Theorem 4 to all Lebesgue-integrable functions $u$.

Remark 6 (Existence of Darling-Kac sets by Aaronson's method). Let us point out that an alternative quick proof of (pointwise dual ergodicity and) the fact that $T$ has Darling-Kac sets can be given by applying Lemma 3.7.4 of [A0] to our reference set $Y=Y(T)$ (compare also [A2]). The advantage of our approach is that it provides us with still more uniform and Darling-Kac sets.

The main ingredient for the proof of Theorems 3 and 4 are the nice ergodic properties common to Markovian piecewise monotonic systems with nonpositive Schwarzian derivative. We are going to exploit these via suitable Markov extensions.

Canonical Markov extensions. Let $(X, T, \xi)$ be a piecewise monotonic system. For $n \geq 0$ define $\mathcal{M}_{n}:=\left\{T^{k} Z: Z \in \xi_{k}, 0 \leq k \leq n\right\}$ and $\mathcal{M}:=$ $\bigcup_{n \geq 0} \mathcal{M}_{n}$. (All members of $\mathcal{M}$ are thus connected sets.) For $B \in \mathcal{M}$, let $\widehat{B}:=$ $\{(x, B): x \in B\}, \widehat{\mathcal{M}}_{n}:=\left\{\widehat{B}: B \in \mathcal{M}_{n}\right\}$ and $\widehat{\mathcal{M}}:=\bigcup_{n \geq 0} \widehat{\mathcal{M}}_{n}$. Finally let $\widehat{X}:=\bigcup_{B \in \mathcal{M}} \widehat{B}=\bigcup \widehat{\mathcal{M}}$. The map $\widehat{T}$ given by $\widehat{T}(x, B):=(T x, T(B \cap \xi(x)))$ is well defined a.e. on $\widehat{X}$, and for $m \geq 1$ we have

$$
\widehat{T}^{m}(x, B)=\left(T^{m} x, T^{m}\left(B \cap \xi_{m}(x)\right)\right)
$$

(cf. [K1]). The natural projection $\pi: \widehat{X} \rightarrow X, \pi(x, B):=x$ is onto and satisfies $\pi \circ \widehat{T}=T \circ \pi$. Letting $\widehat{\xi}:=\widehat{\mathcal{M}} \vee \pi^{-1} \xi$ we obtain a system $(\widehat{X}, \widehat{T}, \widehat{\xi})$ which is Markov by construction and satisfies the definition of a piecewise monotonic system. It is called the canonical Markov extension (CME) of $(X, T, \xi)$. Concerning topology and measures, $\widehat{X}$ will be regarded as the sum of the spaces $\widehat{B}$ for $B \in \mathcal{M}$. One-dimensional Lebesgue measure thereon will again be denoted by $\lambda$. Observe that the image partition $\widehat{T}_{*} \widehat{\xi}$ equals $\widehat{\mathcal{M}}$. If $M$ is some object (point, cylinder, or image interval) belonging to $\widehat{\mathcal{M}}_{n} \backslash \widehat{\mathcal{M}}_{n-1}$, then we say it is on level $n$, and write $\Lambda(M):=n$. Notice that $\Lambda \circ \widehat{T} \leq \Lambda+1$.

Notice also that the construction strongly depends on the choice of $\xi$. It can also change if we refine $\xi_{0}$ by removing a single point from $X$. Therefore it is important to always keep in mind that $\widehat{T}$ does not only depend on $T$ but on the whole ensemble $(X, T, \xi)$. On the other hand, we can systematically exploit this flexibility to choose particularly convenient extensions by refining the partitions $\xi_{0}$ and $\xi$. We will do so in Section 4 below (see also Section 4 of [Z1]). Given two partitions $\eta, \eta^{\prime}$ of $X$, we define $\eta \bowtie \eta^{\prime}$ to be the 
coarsest partition of $X$ into subintervals which refines $\eta$ and $\eta^{\prime}$. In general, this is finer than $\eta \vee \eta^{\prime}$.

The transfer operators $\mathbf{P}$ and $\widehat{\mathbf{P}}$ respectively associated with $T$ and $\widehat{T}$ are also closely related to each other. For $\widehat{u}: \widehat{X} \rightarrow[0, \infty)$ measurable and $\left(\pi_{*} \widehat{u}\right)(x):=\sum_{\pi(\widehat{x})=x} \widehat{u}(\widehat{x})$ we have $\pi_{*}(\widehat{\mathbf{P}} \widehat{u})=\mathbf{P}\left(\pi_{*} \widehat{u}\right) .\left(\pi_{*}\right.$ is just the transfer operator of $\pi$.) Given $u: X \rightarrow[0, \infty)$ we define its lift to the base of $(\widehat{X}, \widehat{T}, \widehat{\xi})$ to be the function $\widehat{u}$ vanishing on $\widehat{X} \cap\{\Lambda \geq 1\}$ for which $\pi_{*} \widehat{u}=u$. Sometimes a convenient way to see that an acim $\widehat{\mu}$ for the CME projects onto an acim $\mu$ of $T$ is to use the fact that

(9) if there is some $Y \subseteq X$ such that $\widehat{\mu}\left(\pi^{-1} Y\right)<\infty$, then $\mu:=\widehat{\mu} \circ \pi^{-1}$ is a $\sigma$-finite acim for $T$,

which is easily seen by inducing on $Y$ and $\pi^{-1} Y$.

Function spaces for Markov systems with nonpositive Schwarzian derivative. As shown in [Mi], the transfer operator of a map with nonpositive Schwarzian derivative preserves certain convenient function spaces. This has been exploited systematically for Markovian situations in $\S 2$ of [K2]. For an open interval $J$ we let $\mathcal{D}(J)$ denote the family of all continuous functions $u: J \rightarrow(0, \infty)$ for which $1 / \sqrt{u}$ is concave, together with the constant function $u=0$. Assume now that $(\widehat{X}, \widehat{T}, \widehat{\xi})$ is a Markov system with nonpositive Schwarzian derivative, and consider the cone $\widehat{\mathcal{D}}:=\left\{\widehat{u} \in \mathcal{C}(\widehat{X}):\left.\widehat{u}\right|_{\widehat{D}} \in \mathcal{D}(\widehat{D})\right.$ for all $\widehat{D} \in \mathcal{M}\}$. Then $(\widehat{X}, \widehat{T}, \widehat{\xi}, \lambda, \widehat{\mathcal{D}})$ is a regular Markov system in the sense of $[\mathrm{K} 2]$, meaning that

$$
\text { if } \widehat{u} \in \widehat{\mathcal{D}} \text { and } \widehat{Z} \in \widehat{\xi}_{k} \text { then } \widehat{\mathbf{P}}^{k}\left(1_{\widehat{Z}} \widehat{u}\right) \in \widehat{\mathcal{D}} \text {, }
$$

$\widehat{\mathcal{D}}$ is closed in the topology of uniform convergence on compact subsets (u.c.s. convergence), $\widehat{\mathcal{D}}-\widehat{\mathcal{D}}$ is dense in $L_{1}(\widehat{X}, \lambda)$,

for all $\widehat{D} \in \mathcal{M}$ and all compact $K \subseteq \widehat{D}$, the set $\left\{\left.\log \widehat{u}\right|_{K}: \widehat{u} \in \widehat{\mathcal{D}}\right\}$ is equicontinuous.

As a consequence,

$$
\text { if } \widehat{u} \in \widehat{\mathcal{D}} \text {, then } \widehat{\mathbf{P}}^{n} \widehat{u} \in \widehat{\mathcal{D}} \cup\{\infty\} \text { for } n \geq 0 .
$$

In particular, Theorem 1 of [K2] applies to our CME, showing that its basic ergodic structure is quite nice.

More on distortion properties of Markov systems with nonpositive Schwarzian derivative. Let us point out that systems of this type also fit into the framework of Markov maps with distortion properties as studied in [A0]. (This also offers an alternative approach to the structure theorem of [K2].) Specifically, 
if $(\widehat{X}, \widehat{T}, \widehat{\xi})$ is a conservative Markov system with nonpositive Schwarzian derivative, then, slightly refining $\widehat{\xi}$, it has the weak distortion property in the sense of $[\mathrm{A} 0, \S 4.3]$.

To see this, assume without loss of generality that $(\widehat{X}, \widehat{T}, \widehat{\xi})$ is conservative ergodic. The higher-order dynamical partitions $\widehat{\xi}_{m}, m \geq 1$, are also Markov for $\widehat{T}$, and we may choose $m$ so large that there is an image interval $B=\widehat{T} V$, $V \in \widehat{\xi}$, which contains a $\delta$-scaled neighbourhood, $\delta>0$, of some cylinder $Z \in$ $\widehat{\xi}_{m}$. Refining the partition once again, we let $\widehat{\eta}:=\widehat{\xi}_{m} \bowtie \widehat{T}^{-1}\left\{Z, Z^{\mathrm{c}}\right\}$, and observe that $(\widehat{X}, \widehat{T}, \widehat{\eta})$ again is a Markov system. Define $\mathfrak{r}:=\left\{W \in \widehat{\eta}_{n}: n \geq 1\right.$, $\left.\widehat{T}^{n} W=Z\right\}$; then, by ergodicity, $\mathfrak{r}$ exhausts our space, $\bigcup \mathfrak{r}=\widehat{X} \bmod \lambda$, and for $W \in \mathfrak{r}$ we obviously have $V \cap \widehat{T}^{-n} W \in \mathfrak{r} \cup\{\emptyset\}$ for any $V \in \widehat{\eta}_{n}, n \geq 1$. Together with the Koebe principle (1) this shows that $\mathfrak{r}$ satisfies the defining properties of a Schweiger collection for $(\widehat{X}, \widehat{T}, \widehat{\eta})(\mathrm{cf}$. $[\mathrm{A} 0, \S 4.3])$, whose existence is asserted by the weak distortion property.

Theorem 4.4.7 of [A0] now ensures that the conservative ergodic system $(\widehat{X}, \widehat{T}, \widehat{\eta})$ is exact if its incidence graph is aperiodic, and hence that in the general case it is the product of an exact system and a finite rotation. It is easily seen that this extends to systems with a dissipative part, so that we find that

any ergodic Markov system $(\widehat{X}, \widehat{T}, \widehat{\xi})$ with nonpositive Schwarzian derivative, not totally dissipative, is exact up to a finite rotation,

which will lead to the corresponding statement for S-unimodal maps.

Below we are going to apply these observations to a CME of our Sunimodal Misiurewicz map T. However, we won't just take the CME of $(X, T, \xi)$ with $\xi=\left\{\left(c_{2}, c\right),\left(c, c_{1}\right)\right\}$. The following trick reveals that in the Misiurewicz case the theorem is an easy corollary of the Markovian results.

Lemma 1 (Towers trivializing Misiurewicz intervals). Let $(X, T, \xi)$ be a piecewise monotonic system and assume that the open interval $I \subseteq X$ is (contained in some $B \in \xi_{0}$ and) measurable with respect to some $\xi_{n}, n \in \mathbb{N}$, and disjoint from the orbits of points from $\partial \xi$. Refine $\xi_{0}$ and $\xi$ by letting $\xi_{0}^{\prime}:=\xi_{0} \bowtie\left\{I, I^{\mathrm{c}}\right\}, X^{\prime}:=\bigcup \xi_{0}^{\prime}$, and $\xi^{\prime}:=\xi_{n} \bowtie T^{-1}\left\{I, I^{\mathrm{c}}\right\}$. Then the CME $\left(\widehat{X^{\prime}}, \widehat{T}, \widehat{\xi^{\prime}}\right)$ of $\left(X^{\prime}, T, \xi^{\prime}\right)$ is trivial above $I$ in that $\pi^{-1} I=\pi^{-1} I \cap\{\Lambda=0\}=$ $I \times\{I\}$.

Proof. Replacing $\xi_{1}$ by $\xi_{n}$ if necessary, we may assume without loss of generality that $n(I)=1 . \xi_{0}^{\prime}$ is the collection of connected components of $X^{\prime}$ and contains $I$. Any (higher-order) cylinder $Z^{\prime} \in \xi_{n}^{\prime}$ is of the form $Z^{\prime}=\bigcap_{k=0}^{n-1} T^{-k} Z_{k}^{\prime}, Z_{k}^{\prime} \in \xi^{\prime}, n \geq 1$, with $Z_{k}^{\prime}=Z_{k} \cap T^{-1} J_{k}$, where $Z_{k} \in \xi$ and $J_{k} \in \xi_{0}^{\prime}$. Since all $J \in \xi_{0}^{\prime}$ are measurable $\xi^{\prime}$, we see that in fact $Z^{\prime}=$ 
$Z \cap T^{-n} J_{n-1}$ with $Z:=\bigcap_{k=0}^{n-1} T^{-k} Z_{k}$. By assumption, however, $T^{n} Z$ either covers $I$ or is disjoint from it, and our assertion follows.

We are now ready to prove the main results of this section.

Proof of Theorems 3 and 4. Recall that by boundedness of $X$ and the fact that $\xi$ generates (cf. (2)), for any $M \in \mathcal{E}(T)$ there is a finite collection $\mathcal{I}$ of open intervals $I \in \mathcal{E}(T)$, each measurable $\xi_{n}$ for some $n \geq 1$, such that $\operatorname{cl}(M) \subset \bigcup \mathcal{I}$. Therefore it is enough to consider the case where $\operatorname{cl}(M)$ is contained in a single open interval $I \in \mathcal{E}(T)$ measurable $\xi_{n}$. We refine $\xi$ as in the preceding lemma, $\xi^{\prime}:=\xi_{n} \bowtie T^{-1}\left\{I, I^{\mathrm{c}}\right\}$, and take a closer look at the CME $\left(\widehat{X^{\prime}}, \widehat{T}, \widehat{\xi^{\prime}}\right)$ of $\left(X^{\prime}, T, \xi^{\prime}\right)$. Theorem 1 of $[\mathrm{K} 2]$ applies to describe the possible types of ergodic behaviour of this Markov extension, and the following arguments are based on its conclusions.

Assume that $\mu(I)>0$ (e.g. $I:=Y=Y(T)$ ). By Theorem 1, $I$ is a sweep-out set for $(X, T)$. In turn, $\widehat{I}:=\pi^{-1} I$ is a sweep-out set for the CME, showing that $\widehat{\mu}(\widehat{I})>0$ for any acim $\widehat{\mu}$ of $\widehat{T}$, hence $\widehat{h}:=d \widehat{\mu} / d \lambda$ is strictly positive on $\widehat{I}$ in this case, since necessarily $\widehat{h} \in \widehat{\mathcal{D}}$ by Theorem 1 of [K2]. As $\widehat{I}$ is recurrent, there is at least one $\widehat{\mu}$, and its restriction $\left.\widehat{\mu}\right|_{\widehat{I}}$ is an acim for the induced map $\widehat{T}_{\widehat{I}}$. The trivial projection $\left.\widehat{\mu}\right|_{\widehat{I}} \circ \pi^{-1}$ therefore is an acim for $T_{I}$ and thus equals (some multiple of) $\left.\mu\right|_{I}$. This shows that $\widehat{\mu}$ is unique up to a constant factor, and hence ergodic. We also see that $h \in \mathcal{D}(I)$ for any interval $I \in \mathcal{E}(T)$, and passing to a slightly larger one which covers $\operatorname{cl}(I)$, we conclude that $h$ is also bounded on $I$. According to (9), we have $\widehat{\mu} \circ \pi^{-1}=\mu$ on $X$ if (from this point on) we normalize $\widehat{\mu}$ appropriately. It is easy to see that since $\pi$ is nonsingular with respect to $\lambda$, exactness of $\widehat{T}^{m}$ implies exactness of $T^{m}$, and applying (16) to $\left(\widehat{X^{\prime}}, \widehat{T}, \widehat{\xi}^{\prime}\right)$, we obtain Theorem 3.

Theorem 1 in [K2] also shows that the cempt $\widehat{T}$ on $(\widehat{X}, \mathcal{B}, \widehat{\mu})$ is pointwise dual ergodic with return sequence given by $\widehat{a}_{I, n}:=\lambda(\widehat{I}) \sum_{k=0}^{n-1} \widehat{\mathbf{P}}^{k} 1_{\widehat{I}}\left(x_{0}\right)$, $n \geq 1$, where $\widehat{x}_{0} \in \widehat{I}$ is fixed. Moreover, although this assertion is not made explicit in the statement of that result, it is shown in its proof that letting $\widehat{U}_{n}:=\sum_{k=0}^{n-1} \widehat{\mathbf{P}}^{k} \widehat{u}, n \geq 1$, we have $\widehat{a}_{I, n}^{-1} \widehat{U}_{n} \rightarrow \lambda(\widehat{u}) \cdot \widehat{h}$ uniformly on compact subsets of $\widehat{X}$ whenever $\widehat{u} \in \widehat{\mathcal{D}} \cap L_{1}(\widehat{X}, \lambda)$. Using the trivial projection $\widehat{I} \rightarrow I$ this immediately implies that $\widehat{a}_{I, n}^{-1} \sum_{k=0}^{n-1} \mathbf{P}^{k} u \rightarrow \lambda(\widehat{u}) \cdot h$ u.c.s. on $I$ for $u:=\pi_{*}(\widehat{u})$. Recalling (10), we see that this applies in particular to $\widehat{\mathbf{P}}^{j} \widehat{u}$ whenever $\widehat{u}$ is the lift of some $\xi_{j}^{\prime}$-simple function $u$ to the base of the tower. Therefore,

$$
\frac{1}{\widehat{a}_{I, n}} \sum_{k=0}^{n-1} \mathbf{P}^{k} u \rightarrow \lambda(u) \cdot h \quad \text { u.c.s. on } I
$$


for such $u$, and since $\xi$ generates, a straightforward sandwich argument enables us to extend this to Riemann-integrable functions $u$ on $X$.

To check that the normalizing sequence $\left(\widehat{a}_{I, n}\right)_{n \geq 1}$ does not depend on $I$, we only have to recall that Proposition 3.7.6 of [A0] ensures that the factor $(X, T, \mu)$ of the pointwise dual ergodic system $\left(\widehat{X^{\prime}}, \widehat{T}, \widehat{\xi^{\prime}}\right)$ is pointwise dual ergodic as well, and has the same return sequence.

If $\mu(I)=0$, we consider the same type of extension $\left(\widehat{X^{\prime}}, \widehat{T}, \widehat{\xi^{\prime}}\right)$ as before, and it is clear that $\widehat{I}=\pi^{-1} I$ belongs to the dissipative part. Hence (as the iterated densities are in $\widehat{\mathcal{D}}$ ), for $\xi_{j}^{\prime}$-simple functions $u, \sum_{k=0}^{n-1} \mathbf{P}^{k} u=$ $\sum_{k=0}^{n-1} \widehat{\mathbf{P}}^{k} \widehat{u}$ is uniformly summable on compact subsets of $I$, implying the assertion in this case.

5. More on the return distribution. Regular variation. We let $T$ be some fixed S-unimodal Misiurewicz map and consider our reference set $Y=Y(T)$ with first return (resp. entrance) time function $\varphi(x):=$ $\inf \left\{n \geq 1: T^{n} x \in Y\right\}, x \in X$. We are interested in finer asymptotic properties of the distribution of $\varphi$ under $\mu_{Y}$. Specifically, we would like to improve the estimates of Proposition 1 and determine the asymptotics of the tail probabilities $\mu_{Y}(Y \cap\{\varphi>n\})$ rather than just their order of magnitude. Of course, the most interesting situation is that of regularly varying tails, which is the very property leading to fine probabilistic properties (cf. Sections 7 and 8 below). Recall that a real-valued function $a$ is regularly varying of index $\varrho \in \mathbb{R}$ at infinity if for any $c>0, \lim _{t \rightarrow \infty} a(c t) / a(t)=c^{\varrho}$ (cf. [BGT]). The collection of functions of this type will be denoted by $\mathcal{R}_{\varrho}$. We shall interpret sequences $\left(a_{n}\right)_{n \geq 1}$ as functions via $t \mapsto a_{[t]}$. Proposition 1 suggests that we can hardly expect the tail to be regularly varying unless $V\left(e^{-t}\right)$ is. The following result shows that this condition is also sufficient if the critical orbit behaves well.

Theorem 5 (Lyapunov exponent and tail of the return distribution). Let $T$ be an S-unimodal Misiurewicz map, let $\mathcal{V}(t):=V\left(e^{-t}\right)$ and assume that $\mathcal{V} \in \mathcal{R}_{-\alpha}$ for some $\alpha>0$ (hence $c$ is flat). If $\left(n^{-1} \log \left|\left(T^{n}\right)^{\prime}\left(c_{1}\right)\right|\right)_{n \geq 1}$ $\in \mathcal{R}_{0}$, then

$$
\mu_{Y}(Y \cap\{\varphi>n\}) \sim h_{Y}(c) \cdot \mathcal{V}\left(\log \left|\left(T^{n}\right)^{\prime}\left(c_{1}\right)\right|\right)
$$

as $n \rightarrow \infty$, where $h_{Y}$ is the normalized invariant density on $Y$. In particular, if the postcritical orbit has a Lyapunov exponent

$$
\lambda_{c}:=\lim _{n \rightarrow \infty} \frac{1}{n} \log \left|\left(T^{n}\right)^{\prime}\left(c_{1}\right)\right| \in(0, \infty),
$$

then

$$
\mu_{Y}(Y \cap\{\varphi>n\}) \sim \lambda_{c}^{-\alpha} h_{Y}(c) \cdot \mathcal{V}(n) .
$$


In the next section we will discuss in which sense existence of $\lambda_{c}$ is typical for Misiurewicz maps. Notice that if $T$ is symmetric about $c$, then $V=2 U^{-1}$, and $\mathcal{V} \in \mathcal{R}_{-\alpha}$ iff its inverse $\mathcal{V}^{-1}(t)=-\log U(t / 2)$ belongs to $\mathcal{R}_{-1 / \alpha}$.

EXAMPLE 5 (Continuation of Example 1). For our standard example, $\mathcal{V}(t) \sim 2[p t / s]^{-\alpha} \in \mathcal{R}_{-\alpha}$ with $\alpha=1 / p$. Hence, whenever $T=T_{a}$ is a Misiurewicz map for which $\lambda_{c}$ exists, then $\mu_{Y}(Y \cap\{\varphi>n\}) \sim 2\left(p \lambda_{c} / s\right)^{-\alpha} h_{Y}(c)$. $n^{-\alpha}$ as $n \rightarrow \infty$.

In the proof of Theorem 5 we will refine the arguments which led to Proposition 1. As a preparation for this (and for the material in the next section), we introduce a few more notations, most of them related to the image process of our system. We let $X$ denote the interval $[a, b]$ which $T$ is initially defined on (so that $T(\partial X) \subseteq \partial X)$. The corresponding natural partition is $\xi=\left\{Z_{0}, Z_{1}\right\}=\{(a, c),(c, b)\}$. Define $\widetilde{D}_{1}:=X$, and let

$$
\widetilde{D}_{n+1}= \begin{cases}T\left(\widetilde{D}_{n}\right) & \text { if } c \notin \widetilde{D}_{n}, \\ T\left(\widetilde{E}_{n}\right) & \text { if } c \in \widetilde{D}_{n},\end{cases}
$$

where $\widetilde{E}_{n}$ is the component of $\widetilde{D}_{n}$ containing $c_{n}$. Then $\widetilde{D}_{n}:=T^{n-1} \xi_{n}\left(c_{1}\right)$ for $n \geq 2$. Similarly, letting $D_{1}:=\left(c_{2}, c_{1}\right) \subseteq \widetilde{D}_{1}$ and, for $n \geq 1$, defining $D_{n+1}:=\left(c_{1}, c_{n+1}\right)$ if $c \in D_{n}$ and $D_{n+1}:=T\left(D_{n}\right)$ otherwise, we have $D_{n} \subseteq$ $\widetilde{D}_{n}, n \geq 1$. (The $\widetilde{D}_{n}$ form the extended Hofbauer tower, while the ordinary Hofbauer tower is given by the $D_{n}$.) Let $J:=T\left(Z_{0} \cap I_{\mathcal{M}}\right) \cap T\left(Z_{1} \cap I_{\mathcal{M}}\right)$, which is a nondegenerate open interval, and $n_{J}:=\min \left\{j \geq 1: c \in T^{j} J\right\}<\infty$. We shall use the easy observation that

if $T$ is Misiurewicz, then each $D_{n}(n \geq 1)$ contains some $T^{j} J, j \in$ $\left\{1, \ldots, n_{J}\right\}$.

The indices $n$ for which $c \in D_{n}$ are called cutting times, and the increasing (and for general unimodal maps possibly terminating) sequence of integers they form is denoted by $\left(S_{k}\right)_{k \geq 0}$ (so that $S_{0}=1$ ). If $S_{k}$ and $S_{k-1}$ exist, the difference $S_{k}-S_{k-1}$ is again a cutting time, and this leads to the definition of the kneading map $Q: \mathbb{N} \rightarrow \mathbb{N} \cup\{\infty\}$ via $S_{k}-S_{k-1}=S_{Q(k)}$ and $Q(k):=\infty$ if there are less than $k$ cutting times. Similarly, the $n$ for which $c \in \widetilde{D}_{n} \backslash D_{n}$ are the consecutive co-cutting times $\widetilde{S}_{k}, k \geq 0$, and differences $\widetilde{S}_{k}-\widetilde{S}_{k-1}$, if well defined, are again cutting times $S_{\widetilde{Q}(k)}$, which defines the co-kneading map $\widetilde{Q}: \mathbb{N} \rightarrow \mathbb{N} \cup\{\infty\}$ with $\widetilde{Q}(k):=\infty$ if there are less than $k$ co-cutting times. We shall use the fact (cf. [B1, p. 98]) that

$$
T \text { is a Misiurewicz map iff } \widetilde{Q} \text { (and hence also } Q \text { ) is bounded. }
$$

The proof of the theorem requires more information about the distortion properties of $T$ : The map $T$ has bounded distortion along the postcritical orbit, and $V$ has bounded distortion on geometrically scaled intervals. 
Lemma 2 (Distortion properties of $T$ ). Let $T$ be an S-unimodal Misiurewicz map.

(a) There is some $\widetilde{\delta}>0$ such that

$$
\left(c_{n}-2 \widetilde{\delta}, c_{n}+2 \widetilde{\delta}\right) \subseteq T^{n} \xi_{n}\left(c_{1}\right) \quad \text { for } n \geq 0 .
$$

In particular, the restrictions of $T^{n}$ to $\xi_{n}\left(c_{1}\right) \cap T^{-n}\left(c_{n}-\widetilde{\delta}, c_{n}+\widetilde{\delta}\right)$ have uniformly bounded distortion by the Koebe principle.

(b) For any $r \in(0,1), V$ has uniformly bounded distortion on intervals of the form $(r t, t)$ with $t>0$.

Proof. (a) We mentioned before (cf. (19)) that the $D_{n}$-part of $\widetilde{D}_{n}$ has a certain positive mimimum length, so that we need only consider the length of the other component $\widetilde{D}_{n} \backslash D_{n}$ of $\widetilde{D}_{n} \backslash\left\{c_{n}\right\}$. It is enough to prove (21) along some subsequence of $\mathbb{N}$ with bounded gaps, since the intermediate steps are then given by a finite number of restricted branches of $T^{i}$ of bounded distortion. The subsequence we use is that of the co-cutting times $\widetilde{S}_{k}$ introduced above, which by (20) has bounded gaps. At these times $n=\widetilde{S}_{k}, \widetilde{D}_{n} \backslash D_{n}$ covers $I_{\mathcal{M}}$, and hence has some definite minimal length.

(b) It is enough to consider the $V_{i}, i \in\{0,1\}$, separately. The distortion of a diffeomorphism between open intervals equals that of its inverse. The inverse of $V_{i}$, defined on some $(0, \eta)$, has nonpositive Schwarzian derivative and hence uniformly bounded distortion on intervals which map into some $(r t, r), t>0$, by the Koebe principle.

Proof of Theorem 5. We are going to show that the tail probabilities are determined by the probabilities to stay close to the postcritical orbit for a long time. Since $T$ itself need not be expanding outside $Y$, we study the shadowing set of $c_{1}$ under a suitable iterate $T^{s}$. For $\varepsilon>0$ and $n \geq 1$ we let $U(n, \varepsilon):=D_{n} \cap\left(c_{n}-\varepsilon, c_{n}+\varepsilon\right)$ be the one-sided $\varepsilon$-neighbourhood of $c_{n}$ in $D_{n}$. Take $K>0$ and $\varrho>1$ as in (3) for $J:=Y$, fix some $s \in \mathbb{N}$ so large that $K \varrho^{s}>2$, and define $\delta_{E}:=\widetilde{\delta}\left(\sup _{X}\left|T^{\prime}\right|\right)^{-s}$ with $\widetilde{\delta}$ as in Lemma 2(a), without loss of generality $\widetilde{\delta}<\operatorname{dist}\left(Y, I_{\mathcal{M}}^{\mathrm{c}}\right)$. For any $n \geq 1$ then,

$$
\begin{aligned}
T^{i} U\left(n, \delta_{E}\right) & \subseteq U(n+i, \widetilde{\delta}) \quad \text { if } 0 \leq i \leq s, \\
U\left(n+s, 2 \delta_{E}\right) & \subseteq T^{s} U\left(n, \delta_{E}\right) \subseteq U(n+s, \widetilde{\delta}) .
\end{aligned}
$$

Letting $Y_{1}^{*}:=U\left(1, \delta_{E}\right)$ and $Y_{n+1}^{*}:=Y_{n}^{*} \cap T^{-n s} U\left(n s, \delta_{E}\right), n \geq 1$, we obtain a decreasing sequence of neighbourhoods of $c_{1}$ in $Y_{0}^{*}:=T Y$. Observe that $Y_{n}^{*}$ is the $n$ - $\delta_{E}$-shadowing set of $c_{1}$ under $T^{s}$, and the first part of (22) shows that the intermediate iterates $T^{k s+i} x, x \in Y_{n}^{*}, 0 \leq i<s$, at least belong to the sets $U(k s+i, \widetilde{\delta})$. Therefore $Y_{0}^{*} \cap\{\varphi \geq n s\} \supseteq Y_{n}^{*}, n \geq 1$, and hence

$$
\mu_{Y}(Y \cap\{\varphi>n s\}) \geq \int_{Y \cap T^{-1} Y_{n}^{*}} h_{Y} d \lambda \sim h_{Y}(c) \cdot V\left(\lambda\left(Y_{n}^{*}\right)\right) \quad \text { as } n \rightarrow \infty .
$$


The upper bound for the tail probabilities requires a bit more work. We are going to prove the following counterpart to $(23)$ : For any $\gamma \in(0,1)$ there are $\widetilde{K}>0$ and $\widetilde{q} \in(0,1)$ such that

$$
\mu_{Y}(Y \cap\{\varphi>n s\}) \leq u_{n}(\gamma)+\widetilde{K} \cdot \widetilde{q}^{n} \quad \text { for } n \geq 1,
$$

where $u_{n}(\gamma):=\mu_{Y}\left(Y \cap T^{-1} Y_{\gamma n}^{*}\right) \sim h_{Y}(c) \cdot V\left(\lambda\left(Y_{\gamma n}^{*}\right)\right)$ as $n \rightarrow \infty$. The differences $E_{n}^{*}:=Y_{n}^{*} \backslash Y_{n+1}^{*}, n \geq 0$, contain the points which escape from the $\delta_{E}$-neighbourhood of the orbit $\left(T^{k s} c_{1}\right)_{k \geq 1}$ at step $n$. Notice also that

$$
T^{n s} E_{n}^{*} \subseteq U(n s, \widetilde{\delta}) \text { is an interval of length larger than } \delta_{E},
$$

since by $(22)$ it contains $U\left(n s, 2 \delta_{E}\right) \backslash U\left(n s, \delta_{E}\right)$. Together with (4) this lower bound for the lengths easily yields a uniform exponential bound on the tails of the conditional entrance-distributions to $Y$ on the $T^{n s} E_{n}^{*}$ : There are $K_{\varphi}>0$ and $q_{\varphi} \in(0,1)$ such that

$$
\lambda_{T^{n s} E_{n}^{*}}\left(T^{n s} E_{n}^{*} \cap\{\varphi \geq j\}\right) \leq K_{\varphi} \cdot q_{\varphi}^{j} \quad \text { for } n, j \geq 1 .
$$

Since $Y_{0}^{*}=Y_{n}^{*} \cup \bigcup_{k=0}^{n-1} E_{k}^{*}$, for $n \geq 1$, we find that for any $\gamma \in(0,1)$,

$$
\begin{aligned}
Y_{0}^{*} \cap\{\varphi \geq n s\} & =Y_{n}^{*} \cup \bigcup_{k=0}^{n-1}\left(E_{k}^{*} \cap\{\varphi \geq n s\}\right) \\
& \subseteq Y_{\gamma n}^{*} \cup \bigcup_{k=0}^{\gamma n}\left(E_{k}^{*} \cap\{\varphi \geq n s\}\right),
\end{aligned}
$$

and we take a closer look at the right-hand union of sets. Observing that $E_{k}^{*} \cap\{\varphi \geq n s\}=E_{k}^{*} \cap T^{-k s}\{\varphi \geq(n-k) s\}$, and recalling (25), we apply the second part of Lemma 2 (a) to the restrictions $\left.T^{k s}\right|_{E_{k}^{*}}, k \geq 1$, to see that there is some $\kappa \geq 1$ such that

$$
\begin{aligned}
\lambda\left(E_{k}^{*} \cap\{\varphi \geq n s\}\right) & \leq \kappa \lambda\left(E_{k}^{*}\right) \lambda_{T^{k s} E_{k}^{*}}\left(T^{k s} E_{k}^{*} \cap\{\varphi \geq(n-k) s\}\right) \\
& \leq \kappa K_{\varphi} \lambda\left(E_{k}^{*}\right) \cdot\left(q_{\varphi}^{s}\right)^{n-k}
\end{aligned}
$$

where the second step uses (26). Our construction furthermore ensures that there is some $r \in(0,1)$ such that

$$
\text { each } E_{n}^{*} \text { is of the form } E_{n}^{*}=\left(c_{n}-t, c_{n}-q t\right) \text { or } E_{n}^{*}=\left(c_{n}+q t, c_{n}+t\right)
$$
with $q \in(r, 1)$ and $t>0$.

Together with Lemma $2(\mathrm{~b})$, this shows that there is some $\widetilde{\kappa} \geq 1$ such that

$$
\begin{aligned}
\mu\left(Y \cap T ^ { - 1 } \left(E_{k}^{*} \cap\right.\right. & \{\varphi \geq n s\})) \\
& \leq \widetilde{\kappa}\left(\sup _{Y} h_{Y}\right) \cdot \lambda\left(Y \cap T^{-1} E_{k}^{*}\right) \cdot \lambda_{E_{k}^{*}}\left(E_{k}^{*} \cap\{\varphi \geq n s\}\right) \\
& \leq \kappa \widetilde{\kappa} K_{\varphi}\left(\sup _{Y} h_{Y}\right) \lambda(Y) \cdot\left(q_{\varphi}^{s}\right)^{n-k} .
\end{aligned}
$$


Therefore, letting $\widetilde{K}:=\kappa \widetilde{\kappa} K_{\varphi}\left(\sup _{Y} h_{Y}\right) \lambda(Y) /\left(1-q_{\varphi}^{s}\right)>0$ and $\widetilde{q}:=q_{\varphi}^{s(1-\gamma)}$ $\in(0,1)$, we have

$$
\sum_{k=0}^{\gamma n} \mu\left(Y \cap T^{-1}\left(E_{k}^{*} \cap\{\varphi \geq n s\}\right)\right) \leq \widetilde{K} \cdot \widetilde{q}^{n} \quad \text { for } n \geq 1 .
$$

Combining this with (27) we obtain (24).

In view of the final statement of Proposition 1, (23) and (24) show that the asymptotic behaviour of $\lambda\left(Y_{n}^{*}\right)$ as $n \rightarrow \infty$ is decisive. As $\delta_{E}<\widetilde{\delta}$ and $Y_{n}^{*}=\left(\left.T^{n s}\right|_{\xi_{n s}\left(c_{1}\right)}\right)^{-1} U\left(n s, \delta_{E}\right)$, the second part of Lemma 2 (a) shows that there is some $D>1$ such that for all $n \geq 1$,

$$
\lambda\left(Y_{n}^{*}\right)=d_{n} \lambda\left(Y_{1}^{*}\right) /\left|\left(T^{n s}\right)^{\prime}\left(c_{1}\right)\right| \quad \text { with } d_{n} \in\left(D^{-1}, D\right),
$$

and hence

$$
V\left(\lambda\left(Y_{n}^{*}\right)\right)=\mathcal{V}\left(\log \left|\left(T^{n s}\right)^{\prime}\left(c_{1}\right)\right|-\log \left(d_{n} \lambda\left(Y_{1}^{*}\right)\right)\right) \sim \mathcal{V}\left(\log \left|\left(T^{n s}\right)^{\prime}\left(c_{1}\right)\right|\right)
$$

as $n \rightarrow \infty$ since $\left(\log \left(d_{n} \lambda\left(Y_{1}^{*}\right)\right)\right)_{n \geq 1}$ is a bounded sequence. From (3) we know that $\log \left|\left(T^{n s}\right)^{\prime}\left(c_{1}\right)\right| \approx n$ and hence $V\left(\lambda\left(Y_{n}^{*}\right)\right) \approx \mathcal{V}(n)$ in any case.

Since the regularly varying function $\mathcal{V}$ has an asymptotic inverse $\mathcal{V}^{-1} \in$ $\mathcal{R}_{-1 / \alpha}$ (cf. [BGT, Theorem 1.5.12]), we conclude that

$$
\left(V\left(\lambda\left(Y_{n}^{*}\right)\right)\right)_{n \geq 1} \in \mathcal{R}_{-\alpha} \quad \text { iff } \quad\left(\log \left|\left(T^{n s}\right)^{\prime}\left(c_{1}\right)\right|\right)_{n \geq 1} \in \mathcal{R}_{1} .
$$

(And $V\left(\lambda\left(Y_{n}^{*}\right)\right.$ ) cannot be regularly varying of a different order.) In this case, taking $\gamma$ arbitrarily close to 1 , we find that

$$
\mu_{Y}(Y \cap\{\varphi>n\}) \sim h_{Y}(c) \cdot \mathcal{V}\left(\log \left|\left(T^{n}\right)^{\prime}\left(c_{1}\right)\right|\right)
$$

(since the contributions $\log \left|\left(T^{i}\right)^{\prime}\left(c_{n s}\right)\right|, 0<i<s$, of the intermediate steps are bounded.)

6. Existence of the postcritical Lyapunov exponent. The purpose of the present section is to show that in a reasonable sense "most" Misiurewicz maps from a full family have a postcritical Lyapunov exponent $\lambda_{c}$. Let $T$ be a unimodal map. Recall that the itinerary of the point $x$ is the sequence $\nu(x)=\left(\nu(x)_{i}\right)_{i \geq 1} \in\{0, C, 1\}^{\mathbb{N}}$ with $\nu(x)_{i}=0$ if $T^{i-1} x<c, \nu(x)_{i}=C$ if $T^{i-1} x=c$, and $\nu(x)_{i}=1$ if $T^{i-1} x>c$. Most important, the kneading invariant of $T$ is the itinerary of its critical value $c_{1}, \nu(T):=\nu\left(c_{1}\right)$. In any case, $\nu(T)_{0}=1$ and $\nu(T)_{1}=0$ unless the dynamics is trivial. There are various characterizations of those admissible sequences $\nu \in\{0,1\}^{\mathbb{N}}$ which actually occur as the kneading invariant of some unimodal map, and we shall use a criterion from [B1], [B2] based on the kneading maps.

Given any symbol sequence $\nu=\nu_{1} \nu_{2} \ldots \in\{0,1\}^{\mathbb{N}}$ starting with 1 , there is a well defined splitting beginning at the second digit:

$$
\nu=1 \triangle_{1} \triangle_{2} \triangle_{3} \ldots
$$


where each $\triangle_{j}$ is a basic block, meaning that it agrees with an initial segment of $\nu$ except for the last symbol, i.e. $\triangle_{i}=e_{1} \ldots e_{m-1} e_{m}=\nu_{1} \ldots \nu_{m-1} \nu_{m}^{\prime}$, where $\nu_{m}^{\prime}=\nu_{m}+1 \bmod 2$. Letting $\kappa:=\min \left\{i \geq 1: \nu_{i}=1\right\}$, we obtain the co-splitting of $\nu$ which starts at the $\kappa+1$ st digit:

$$
\nu=10 \ldots 01 \widetilde{\triangle}_{1} \widetilde{\triangle}_{2} \widetilde{\triangle}_{3} \ldots,
$$

where again the $\widetilde{\triangle}_{j}$ are basic blocks. Based on this construction, we define the cutting and co-cutting times of $\nu$ by $S_{0}:=1, S_{k}:=S_{0}+\sum_{j=1}^{k}\left|\triangle_{j}\right|$, and $\widetilde{S}_{0}:=\kappa, \widetilde{S}_{k}:=\widetilde{S}_{0}+\sum_{j=1}^{k}\left|\widetilde{\triangle}_{j}\right|$, where for any block $\square$ of symbols we let $|\square|$ denote its length. Bruin's admissibility condition now states that

$\nu$ is admissible iff the differences $S_{k}-S_{k-1}$ and $\widetilde{S}_{k}-\widetilde{S}_{k-1}$ of consecutive cutting and co-cutting times are again cutting times,

in which case the kneading and co-kneading maps $Q, \widetilde{Q}: \mathbb{N} \rightarrow \mathbb{N}$ of $\nu$ are well defined by $S_{k}-S_{k-1}=S_{Q(k)}$ and $\widetilde{S}_{k}-\widetilde{S}_{k-1}=S_{\widetilde{Q}(k)}, k \geq 1$, and agree with the (co-)kneading maps of any unimodal map $T$ with $\nu(T)=\nu$ (and the same is true for the (co-)cutting times). Recalling (20), we call an admissible $\nu \in\{0,1\}^{\mathbb{N}}$ a Misiurewicz kneading sequence if $\widetilde{Q}$ is bounded, which is equivalent to $\sup _{j \geq 1}\left|\widetilde{\triangle}_{j}\right|<\infty$.

Proposition 2 (For typical Misiurewicz kneadings $\lambda_{c}$ exists). Let $\mu_{\sigma}$ be any invariant Borel probability measure for the shift $\sigma$ on $\{0,1\}^{\mathbb{N}}$. If the Misiurewicz kneading sequence $\nu$ is a typical point for $\mu_{\sigma}$, then $\lambda_{c}$ exists for any $S$-unimodal map $T$ with kneading invariant $\nu$.

Proof. Fix some $T$ with $\nu(T)=\nu$. As $\nu$ is typical for $\mu_{\sigma}$, its orbit $O(\nu):=$ $\left(\sigma^{n} \nu\right)_{n \geq 0}$ is dense in the closed subshift $\Omega:=\operatorname{supp}\left(\mu_{\sigma}\right) \subseteq\{0,1\}^{\mathbb{N}}$. We lift the potential $\log \left|T^{\prime}\right|$ to $\Omega$ by first defining it on the orbit via $\psi\left(\sigma^{n} \nu\right):=$ $\log \left|T^{\prime}\left(c_{n+1}\right)\right|, n \geq 0$. Now $\left.\psi\right|_{O(\nu)}$ is easily seen to be uniformly continuous and hence extends to a unique uniformly continuous function $\psi$ on $\Omega$. Since $\nu$ is typical for $\mu_{\sigma}$, we have

$$
\lim _{n \rightarrow \infty} \frac{1}{n} \sum_{k=0}^{n-1} \psi\left(\sigma^{n} \nu\right)=\lim _{n \rightarrow \infty} \frac{1}{n} \log \left|\left(T^{n}\right)^{\prime}\left(c_{1}\right)\right|=\mu_{\sigma}(\psi) .
$$

It remains to check that the concept of being typical for some shiftinvariant probability is not void inside the comparatively small collection of Misiurewicz kneading sequences. We do so by showing that the latter essentially contains (for example) the golden mean subshift $\Sigma:=\left\{\omega \in\{0,1\}^{\mathbb{N}}: \omega\right.$ does not contain a block of two or more zeros $\}$, which has positive topological entropy $h_{\text {top }}\left(\left.\sigma\right|_{\Sigma}\right)=\log ((1+\sqrt{5}) / 2)$. Hence there is a rich supply of invariant measures of positive entropy that live on Misiurewicz sequences. 
Proposition 3 (Golden mean subshift gives Misiurewicz kneadings). For any $\omega \in \Sigma$,

$$
\nu:=100111 \omega
$$

defines a Misiurewicz kneading sequence.

Proof. From the given initial block $\nu_{1} \ldots \nu_{6}=100111$ we read off that $S_{0}=1, S_{1}=2, S_{2}=3, \kappa=\widetilde{S}_{0}=4, \triangle_{1}=\triangle_{2}=0$, and $\triangle_{3}=\widetilde{\triangle}_{1}=11$, so that $\nu_{1} \ldots \nu_{6}=1 \triangle_{1} \triangle_{2} \triangle_{3} 1=1001 \widetilde{\triangle}_{1}$ in compliance with (30). To prove our claim, we show inductively that whenever we add blocks of the form $\square=1$ or $\square=01$ to our initial piece (which produces all sequences in $\Sigma$ ), we obtain a sequence $\nu$ whose basic blocks $\triangle_{i}$ and $\widetilde{\triangle}_{j}$ from the (co-)splitting above belong to $\{0,11,101\}=: \mathfrak{B}$ so that $\nu$ has bounded kneading and co-kneading maps.

Assume then that for some $n \geq 6$,

$$
\nu_{1} \ldots \nu_{n}=1 \triangle_{1} \ldots \triangle_{i} 1=1001 \widetilde{\triangle}_{1} \ldots \widetilde{\triangle}_{j}
$$

with basic blocks from $\mathfrak{B}$. Adjoining $\square=1$ we obtain

$$
\nu_{1} \ldots \nu_{n} \nu_{n+1}=1 \triangle_{1} \ldots \triangle_{i} \triangle_{i+1}=1001 \widetilde{\triangle}_{1} \ldots \widetilde{\triangle}_{j} 1
$$

with $\triangle_{i+1}=11 \in \mathfrak{B}$. Similarly, if instead we add $\square=01$, we get

$$
\nu_{1} \ldots \nu_{n} \nu_{n+1} \nu_{n+2}=1 \triangle_{1} \ldots \triangle_{i} \triangle_{i+1}=1001 \widetilde{\triangle}_{1} \ldots \widetilde{\triangle}_{j} \widetilde{\triangle}_{j+1} 1
$$

with $\triangle_{i+1}=101$ and $\widetilde{\triangle}_{j+1}=0$ both in $\mathfrak{B}$. In any case, we are again in the situation of (31) with the roles of splitting and co-splitting interchanged, and we can continue with our construction.

7. More on limit distributions if $\mu$ is finite. Regular variation often is the key to questions about limit distributions and enables us to go beyond the regime of the classical CLT. Again we consider occupation times of the reference set $Y=Y(T)$, thus asking for the asymptotic distributional behaviour of the ergodic sums

$$
\mathbf{S}_{n}\left(1_{Y}\right)=\sum_{k=0}^{n-1} 1_{Y} \circ T^{k}, \quad n \geq 1 .
$$

By now we are prepared to expect results analogous to those we have for maps with indifferent fixed points, and we will in fact see that the same method as in [Z2] applies to provide us with stable limit theorems. To formulate the result, we need to recall the limit laws we have to expect.

A real-valued random variable $R$ (respectively its distribution) is called stable (cf. [AD1] or [IL]) if for all $a, b>0$ there are $c>0$ and $d \in \mathbb{R}$ such that $a R+b R^{*} \stackrel{\mathrm{d}}{=} c R+d$, where $R^{*}$ is an independent copy of $R$ and $R \stackrel{\mathrm{d}}{=} S$ means equality of distributions. In this case $a^{\alpha}+b^{\alpha}=c^{\alpha}$ for some $\alpha \in(0,2]$, called 
the order of $R$. Up to translation and scaling, any stable random variable of order $\alpha \in(1,2]$, which is the case of interest to us, belongs to the family $\left(\Xi_{\alpha, \beta}\right)_{\alpha \in(1,2], \beta \in[-1,1]}$ of variables, indexed by the order $\alpha$ and the skewness parameter $\beta$, and uniquely determined by their characteristic functions

$$
\mathbb{E}\left[e^{i t \Xi_{\alpha, \beta}}\right]=e^{-|t|^{\alpha}(1-i \beta \operatorname{sgn}(t) \tan (\alpha \pi / 2)) / 2} .
$$

(For $\alpha=2$ this is the standard normal distribution $\mathcal{N}(0,1)$.) The following is the counterpart to the CLT we obtained in the case $\alpha>2$ as a consequence of the rate of mixing (cf. Theorem 2).

Theorem 6 (Stable limit distributions for occupation times). Let $T$ be an S-unimodal Misiurewicz map for which $\lambda_{c}:=\lim _{n \rightarrow \infty} n^{-1} \log \left|\left(T^{n}\right)^{\prime}\left(c_{1}\right)\right|$ $\in(0, \infty)$ exists.

(a) If $\mathcal{V}(t):=V\left(e^{-t}\right) \in \mathcal{R}_{-2}$ and $\int_{1}^{\infty} \mathcal{V}(\sqrt{t}) d t=\infty$, then there is some $B \in \mathcal{R}_{1 / 2}$ such that

$$
\frac{1}{B(n)}\left(\sum_{k=0}^{n-1} 1_{Y} \circ T^{k}-n \mu(Y)\right) \stackrel{\mathcal{L}(\lambda)}{\Longrightarrow} \mu(Y) \cdot \mathcal{N}(0,1),
$$

where $B$ is specified by $t \ell(B(t)) \sim B(t)^{2}$ with $\ell(t):=\lambda_{c}^{-2} h(c)$. $\int_{1}^{t} \mathcal{V}(\sqrt{s}) d s$

(b) If $\mathcal{V}(t):=V\left(e^{-t}\right) \in \mathcal{R}_{-\alpha}$ for some $\alpha \in(1,2)$, then there is some $B \in \mathcal{R}_{1 / \alpha}$ such that

$$
\frac{1}{B(n)}\left(\sum_{k=0}^{n-1} 1_{Y} \circ T^{k}-n \mu(Y)\right) \stackrel{\mathcal{L}(\lambda)}{\Longrightarrow} \mu(Y) \cdot \Xi_{\alpha, 1} .
$$

Here $B(t):=-\Gamma(1-\alpha) \cos (\alpha \pi / 2) h(c)^{1 / \alpha} \lambda_{c}^{-1} \cdot \mathcal{V}^{-1}(1 / t)$ with $\mathcal{V}^{-1} \in$ $\mathcal{R}_{-1 / \alpha}$ asymptotically inverse to $\mathcal{V}$.

ExAmPle 6 (Continuation of Example 1). Recall that $\mathcal{V}(t) \sim 2[p t / s]^{-\alpha}$ as $t \rightarrow \infty$.

(a) If $p=1 / 2$, then $\int_{1}^{t} \mathcal{V}(\sqrt{s}) d s \sim 2(s / p)^{2} \log t \rightarrow \infty$, and the theorem applies with $\ell(t)=2 h(c)\left[s /\left(p \lambda_{c}\right)\right]^{2} \cdot \log t$ and $B(t)=\left[(s \sqrt{h(c)}) /\left(p \lambda_{c}\right)\right]$. $\sqrt{t \log t}$.

(b) If $p \in(1 / 2,1)$, then (34) holds with $B(t)=-\Gamma(1-\alpha) \cos (\alpha \pi / 2)$. $(2 h(c))^{1 / \alpha}\left[s /\left(p \lambda_{c}\right)\right] \cdot t^{1 / \alpha}$.

Proof of Theorem 6. We use the same device as in [Z2] and consider the centred observable $f^{\mathrm{c}}:=1_{Y}-\mu(Y)$ and its induced version $f_{Y}^{\mathrm{c}}: Y \rightarrow \mathbb{R}$, $f_{Y}^{\mathrm{c}}:=\sum_{k=0}^{\varphi-1} f^{\mathrm{c}} \circ T^{k}$. Notice that $f_{Y}^{\mathrm{c}}=1-\mu(Y) \varphi$, which is bounded above and by Theorem 5 satisfies

$$
\mu_{Y}\left(\left\{f_{Y}^{\mathrm{c}}<-n\right\}\right) \sim \mu_{Y}(\{\varphi>n / \mu(Y)\}) \sim\left(\mu(Y) / \lambda_{c}\right)^{\alpha} h_{Y}(c) \cdot \mathcal{V}(n) .
$$


By Remark 2, $\left(Y, T_{Y}, \xi_{Y}\right)$ is a Gibbs-Markov map in the sense of [A0], [AD1], and [AD2], and clearly $\varphi$ and $f_{Y}^{\mathrm{c}}$ are measurable $\xi_{Y}$.

(a) Divergence of $\int_{1}^{\infty} \mathcal{V}(\sqrt{t}) d t$ just means that $\int_{Y} \varphi^{2} d \mu_{Y}=\infty$, and by regular variation of the tail, the distributions of $\varphi$ and $f_{Y}^{c}$ are in the "nonnormal" domain of attraction of the normal law (cf. [IL, Theorem 2.6.2]). Applying the last corollary of [AD2], we see that

$$
\frac{1}{B(n / \mu(Y))} \sum_{k=0}^{n-1} f_{Y}^{\mathrm{c}} \circ T_{Y}^{k} \stackrel{\mu_{Y}}{\Longrightarrow} \mu(Y) \cdot \mathcal{N}(0,1),
$$

with $B$ as in the statement of the theorem. Proposition 2 of [Z2] now gives

$$
\frac{1}{B(n)} \sum_{k=0}^{n-1} f^{\mathrm{c}} \circ T^{k} \stackrel{\mathcal{L}(\mu)}{\Longrightarrow} \mu(Y) \cdot \mathcal{N}(0,1),
$$

which easily extends to $\stackrel{\mathcal{L}(\lambda)}{\Longrightarrow}$ convergence, thus proving (33).

(b) Theorem 6.1 of [AD1] applies and provides us with a normalizing function $B \in \mathcal{R}_{1 / \alpha}$ such that

$$
\frac{1}{B(n / \mu(Y))} \sum_{k=0}^{n-1} f_{Y}^{c} \circ T_{Y}^{k} \stackrel{\mu_{Y}}{\Longrightarrow} \mu(Y) \cdot \Xi_{\alpha, 1},
$$

where $B$ is as specified. Using Proposition 2 of [Z2] again, we end up with (34).

8. More on wandering rates and limit distributions if $\mu$ is infinite. In the infinite measure preserving case, too, regular variation of return distributions is crucial for strong stochastic properties to hold. First of all, it enables us to determine the asymptotics of wandering rates and return sequences.

Recall (see e.g. Section 3.8 of [A0]) that the wandering rate of a set $E \in \mathcal{B}, 0<\mu(E)<\infty$, under a cempt $T$ is the sequence defined by $w_{n}(E):=\mu\left(\bigcup_{k=0}^{n-1} T^{-k} E\right)=\sum_{k=0}^{n-1} \mu\left(E \cap\left\{\varphi_{E} \geq k\right\}\right), n \geq 1$. The wandering rate depends on $E$, and, given $T$, there are no sets with maximal rate. Still, $T$ may have sets $E$ with minimal wandering rate, meaning that $\varliminf_{n \rightarrow \infty} w_{n}(Z) / w_{n}(E) \geq 1$ for all $Z \in \mathcal{B}, 0<\mu(Z)<\infty$. If this is the case, we let $\mathcal{W}(T) \subseteq \mathcal{A}$ denote the collection of sets which have minimal wandering rate under $T$, and simply write $\left(w_{n}(T)\right)_{n \geq 1}$ for any sequence representing the asymptotic equivalence class of such $\left(w_{n}(E)\right)$, which is then referred to as the wandering rate of $T$.

Theorem 7 (Wandering rates and return sequences). Suppose $T$ is an S-unimodal Misiurewicz map for which $\lambda_{c}:=\lim _{n \rightarrow \infty} n^{-1} \log \left|\left(T^{n}\right)^{\prime}\left(c_{1}\right)\right| \in$ $(0, \infty)$ exists, and assume that $\mathcal{V}(t):=V\left(e^{-t}\right) \in \mathcal{R}_{-\alpha}$ for some $\alpha \in(0,1]$. 
Then all $E \in \mathcal{E}(T)$ have minimal wandering rate, and

$$
w_{n}(T) \sim \lambda_{c}^{-\alpha} h(c) \sum_{k=0}^{n-1} \mathcal{V}(k) \in \mathcal{R}_{1-\alpha},
$$

with $\sum_{k=0}^{n-1} \mathcal{V}(k) \sim n \mathcal{V}(n) /(1-\alpha)$ in case $\alpha<1$. Moreover, the return sequence of $T$ satisfies $a_{n}(T) \sim[\Gamma(2-\alpha) \Gamma(1+\alpha)]^{-1} \cdot n / w_{n}(T) \in \mathcal{R}_{\alpha}$.

EXAmple 7 (Continuation of Example 1). Recalling that $\mathcal{V}(t) \sim$ $2[p t / s]^{-\alpha}$, we find that

$$
w_{n}(T) \sim 2 h(c)\left(\frac{s}{p \lambda_{c}}\right)^{\alpha} \cdot \begin{cases}\log n & \text { if } p=1 \\ n^{1-\alpha} /(1-\alpha) & \text { if } p>1\end{cases}
$$

and

$$
a_{n}(T) \sim \frac{2 h(c)}{\Gamma(2-\alpha) \Gamma(1+\alpha)}\left(\frac{s}{p \lambda_{c}}\right)^{\alpha} \cdot \begin{cases}n / \log n & \text { if } p=1, \\ (1-\alpha) n^{-\alpha} & \text { if } p>1 .\end{cases}
$$

Proof. Consider $Y=Y(T)$. By Theorem 5, $w_{n}(Y)=\sum_{k=0}^{n-1} \mu(Y \cap$ $\{\varphi>n\}) \sim \lambda_{c}^{-\alpha} h(c) \sum_{k=0}^{n-1} \mathcal{V}(k)$. By Propositions 1.5.8 and 1.5.9(a) of [BGT], the rightmost term always belongs to $\mathcal{R}_{1-\alpha}$, and $\sum_{k=0}^{n-1} \mathcal{V}(k) \sim n \mathcal{V}(n) /(1-\alpha)$ if $\alpha<1$. Since, moreover, $Y$ is a uniform set for $T$ (cf. Corollary 1), Proposition 3.8.7 of [A0] immediately gives the asymptotics of $\left(a_{n}(T)\right)_{n \geq 1}$. Now Theorem 3.8.3 of [A0] ensures that all uniform sets $Y$ have the same (minimal) wandering rate. (Notice that although this assertion is not stated there, Theorem 3.8.3 of [A0] actually requires $\left(a_{n}(T)\right)$ to be regularly varying; see the corrections to $[\mathrm{A} 0]$.

Without any effort, we can now apply Corollary 3.7.3 of [A0] to obtain

Theorem 8 (Darling-Kac theorem). Let $T$ be an S-unimodal Misiurewicz map for which $\lambda_{c}:=\lim _{n \rightarrow \infty} n^{-1} \log \left|\left(T^{n}\right)^{\prime}\left(c_{1}\right)\right| \in(0, \infty)$ exists, and assume that $\mathcal{V}(t):=V\left(e^{-t}\right) \in \mathcal{R}_{-\alpha}$ for some $\alpha \in(0,1]$. Then for any $f \in L_{1}(\mu)$ with $\mu(f) \neq 0$,

$$
\frac{1}{a_{n}(T)} \mathbf{S}_{n}(f)=\frac{1}{a_{n}(T)} \sum_{k=0}^{n-1} f \circ T^{k} \stackrel{\mathcal{L}(\lambda)}{\Longrightarrow} \mu(f) \cdot W^{(\alpha)},
$$

where $W^{(\alpha)}$ is a random variable having the normalized Mittag-Leffler distribution of order $\alpha$, that is,

$$
\mathbf{E}\left[e^{z W^{(\alpha)}}\right]=\sum_{m \geq 0} \frac{\Gamma(1+\alpha)^{m}}{\Gamma(1+m \alpha)} z^{m} .
$$

Again, this applies to the Misiurewicz maps with $\lambda_{c} \in(0, \infty)$ from the family of Example 1 (with the normalizations given in Example 7), and $f:=1_{Y}, Y=Y(T)$, thus completing the picture of the asymptotic behaviour of occupation times of $Y$ for the whole parameter range $p \in \mathbb{R}_{+}$. 
REMARK 7 (Dynkin-Lamperti arcsin law). As another immediate probabilistic consequence of Theorem 7, let us mention that the Dynkin-Lamperti type arcsin law of Theorem 1 of [T4] applies to sets $A \in \mathcal{E}(T)$ for any S-unimodal Misiurewicz map $T$ for which $\lambda_{c} \in(0, \infty)$ exists and $\mathcal{V}(t):=$ $V\left(e^{-t}\right) \in \mathcal{R}_{-\alpha}$ for some $\alpha \in(0,1]$.

Acknowledgments. I am indebted to G. Keller for stimulating and enlightening discussions. I am also grateful to M. Holland, O. Jenkinson, C. Liverani, and M. Thaler for motivating conversations. This research was supported by the Austrian Science Foundation FWF, project P14734-MAT. Part of this work was done during a visit to the SNS in Pisa.

\section{References}

[A0] J. Aaronson, An Introduction to Infinite Ergodic Theory, Math. Surveys Monogr. 50, Amer. Math. Soc., 1997.

[A1] - , The asymptotic distributional behaviour of transformations preserving infinite measures, J. Anal. Math. 39 (1981), 203-234.

[A2] -, Random f-expansions, Ann. Probab. 14 (1986), 1037-1057.

[AD1] J. Aaronson and M. Denker, Local limit theorems for partial sums of stationary sequences generated by Gibbs-Markov maps, Stochast. Dynam. 1 (2001), 193-238.

[AD2] - - - A local limit theorem for stationary processes in the domain of attraction of a normal distribution, in: Asymptotic Methods in Probability and Statistics with Applications (St. Petersburg, 1998), N. Balakrishnan, I. A. Ibragimov and V. B. Nevzorov (eds.), Birkhäuser, 2001, 215-223.

[BM] M. Benedicks and M. Misiurewicz, Absolutely continuous invariant measures for maps with flat tops, Inst. Hautes Études Sci. Publ. Math. 69 (1989), 203-213.

[BGT] N. H. Bingham, C. M. Goldie and J. L. Teugels, Regular Variation, Cambridge Univ. Press, 1989.

[B1] H. Bruin, Invariant measures of interval maps, PhD thesis, Delft, 1994.

[B2] - Combinatorics of the kneading map, Internat. J. Bifur. Chaos Appl. Sci. Engrg. 5 (1995), 1339-1349.

[Ho] M. Holland, Slowly mixing systems and intermittency maps, preprint, 2002.

$[\mathrm{Hu}] \mathrm{H} . \mathrm{Hu}$, Decay of correlations for piecewise smooth maps with indifferent fixed points, preprint.

[IL] I. A. Ibragimov and Yu. V. Linnik, Independent and Stationary Sequences of Random Variables, translated from Russian and edited by J. F. C. Kingman, WoltersNoordhoff, Groningen, 1971.

[K1] G. Keller, Lifting measures to Markov extensions, Monatsh. Math. 108 (1989), 183-200.

[K2] G. Keller, Exponents, attractors, and Hopf decompositions for interval maps, Ergodic Theory Dynam. Systems 10 (1990), 717-744.

[LSV] C. Liverani, B. Saussol and S. Vaienti, A probabilistic approach to intermittency, ibid. 19 (1999), 671-686.

[Mi] M. Misiurewicz, Absolutely continuous measures for certain maps of an interval, Inst. Hautes Études Sci. Publ. Math. 53 (1981), 17-51.

[Sa] O. Sarig, Subexponential decay of correlations, Invent. Math. 150 (2002), 629-653. 
[T1] M. Thaler, Estimates of the invariant densities of endomorphisms with indifferent fixed points, Israel J. Math. 37 (1980), 303-314.

[T2] -, Transformations on [0,1] with infinite invariant measures, ibid. 46 (1983), 6796.

[T3] - A limit theorem for the Perron-Frobenius operator of transformations on $[0,1]$ with indifferent fixed points, ibid. 91 (1995), 111-127.

[T4] - The Dynkin-Lamperti arc-sine laws for measure preserving transformations, Trans. Amer. Math. Soc. 350 (1998), 4593-4607.

[Th] H. Thunberg, Positive exponent in families with flat critical point, Ergodic Theory Dynam. System 19 (1999), 767-807.

[Yo] L. S. Young, Recurrence times and rates of mixing, Israel J. Math. 110 (1999), 153-188.

[Z1] R. Zweimüller, Ergodic properties of infinite measure preserving interval maps with indifferent fixed points, Ergodic Theory Dynam. Systems 20 (2000), 1519-1549.

[Z2] - Stable limits for probability preserving maps with indifferent fixed points, Stochast. Dynam. 3 (2003), 83-99.

Mathematics Department

Imperial College London

180 Queen's Gate

London SW7 2AL, UK

E-mail: r.zweimueller@imperial.ac.uk

Received 17 December 2002;

in revised form 8 March 2004 